\title{
Quantificação de citocinas no conteúdo abomasal de bovinos de corte na presença ou ausência de úlceração gástrica
}

Fernando Christiano Gabriel Morelli[a]", Leonardo Aparecido Teixeira Bentin ${ }^{[a]}$, Priscila Dalmagro ${ }^{[a]}$, Gisele Fabrino Machado[a], Silvia Helena Venturoli Perri[b], Francisco Leydson Formiga Feitosa ${ }^{\left[{ }^{[a]}\right.}$, Luiz Claudio Nogueira Mendes ${ }^{[a]}$, Juliana Regina Peiró[a]

\footnotetext{
[a] Departamento de Clínica, Cirurgia e Reprodução Animal, Faculdade de Medicina Veterinária, Universidade Estadual Paulista (UNESP), Araçatuba, SP, Brasil

[b] Departamento de Apoio, Produção e Saúde Animal, Faculdade de Medicina Veterinária, Universidade Estadual Paulista (UNESP), Araçatuba, SP, Brasil
}

*Autor correspondente

e-mail: fcgmorelli@yahoo.com.br

\section{Resumo}

O manejo intensivo na busca de aumentos de produtividade leva à frequência das doenças do sistema digestivo de bovinos. Úlceras abomasais estão presentes em animais de todas as idades e raças, mas são mais comuns em animais em manejo intensivo. Úlceras e erosões são achados comuns no abomaso dos bovinos e são classificadas em grau 1-4. Informações sobre a resposta do sistema imune na mucosa do aparelho gastrointestinal da maioria das espécies animais. A resposta do sistema imune no conteúdo abomasal não foram encontradas informações. Portanto, o objetivo do estudo foi quantificar citocinas no conteúdo abomasal de bovinos de corte na presença ou ausência de úlceras. Os abomasos foram colhidos em um abatedouro supervisionado pelo SIF. Foram selecionados 18 abomasos, machos e fêmeas, com idade entre 3,5 e 4 anos, alimentados a pasto e em confinamento, classificadas macroscopicamente e por histopatologia descrita anteriormente (Hund, 2015). As concentrações de TNF- $\alpha$, IL-2, IL-6 e IFN- $\gamma$ (Th1), IL-4, IL-10 (Th2) e IL-17A (Th17) foram mensuradas nas amostras de líquido abomasal pelo método Cytometric Bead Array (CBA) Human Th1/Th2/Th17 Cytokine Kit. Para justificar a utilização do kit humano foi realizado um "BLAST" (Bos taurus). Após análise de variância, os dados foram transformados em log $(\mathrm{x}+1)$ e as médias foram comparadas pelo teste de Kruskal-Wallis $(\mathrm{P}<0,05)$. Os abomasos foram avaliados macroscopicamente e por histopatologia, para verificar presença ou ausência de úlceras (erosões superficiais ou erosões mais profundas, com sangramento e necrose tecidual) da região cárdica. Os valores das citocinas TNF- $\alpha$, IL-2, IL-6, IFN- $\gamma$, IL-4 e IL-10, encontrados nos líquidos abomasais dos animais nos diferentes grupos foram semelhantes. Concentrações de IL-17A não foram detectadas (dados não mostrados). As concentrações 
das citocinas foram determinadas no líquido abomasal e os valores foram semelhantes entre os grupos, indicando que não houve um incremento na liberação dessas citocinas em virtude das ulcerações, havendo um comportamento de equilíbrio entre a resposta pró-inflamatória e anti-inflamatória. Respostas inflamatórias do sistema imune inato podem ser iniciadas, mantidas ou aumentadas nos estômagos de bovinos. A presença de neovascularização e tecido de granulação, assim como células inflamatórias com presença de neutrófilos e de células mononucleares, indicam processo cicatricial. 0 comportamento das citocinas no líquido abomasal em nosso estudo foi semelhante ao observado nos estômagos de bovinos. Portanto, sugere-se que a cicatrização das lesões observadas nos abomasos avaliados ocorreu sem a necessidade de uma resposta inflamatória local aumentada. As citocinas foram detectadas no líquido abomasal e não houve aumento da liberação das citocinas no líquido, indicando um comportamento equilibrado entre os perfis Th1 e Th2 na resposta imune em relação às úlceras da região cárdica. 